\title{
Comparing Supervised Exercise Therapy to Invasive Measures in the Management of Symptomatic Peripheral Arterial Disease
}

\author{
Thomas Aherne, ${ }^{1}$ Seamus McHugh, ${ }^{1}$ Elrasheid A. Kheirelseid, ${ }^{1}$ Michael J. Lee, ${ }^{2}$ \\ Noel McCaffrey, ${ }^{3}$ Daragh Moneley, ${ }^{1}$ Austin L. Leahy, ${ }^{1}$ and Peter Naughton ${ }^{1}$ \\ ${ }^{1}$ Department of Vascular Surgery, Beaumont Hospital, Dublin 9, Ireland \\ ${ }^{2}$ Department of Interventional Radiology, Beaumont Hospital, Dublin 9, Ireland \\ ${ }^{3}$ Department of Human and Health Performance, Dublin City University, Dublin 9, Ireland \\ Correspondence should be addressed to Thomas Aherne; thomasaherne@rcsi.ie
}

Received 1 June 2015; Revised 5 October 2015; Accepted 11 October 2015

Academic Editor: Miltiadis I. Matsagkas

Copyright (C) 2015 Thomas Aherne et al. This is an open access article distributed under the Creative Commons Attribution License, which permits unrestricted use, distribution, and reproduction in any medium, provided the original work is properly cited.

\begin{abstract}
Peripheral arterial disease (PAD) is associated with considerable morbidity and mortality. Consensus rightly demands the incorporation of supervised exercise training (SET) into PAD treatment protocols. However, the exact role of SET particularly its relationship with intervention requires further clarification. While supervised exercise is undoubtedly an excellent tool in the conservative management of mild PAD its use in more advanced disease as an adjunct to open or endovascular intervention is not clearly defined. Indeed its use in isolation in this cohort is incompletely reported. The aim of this review is to clarify the exact role of SET in the management of symptomatic PAD and in particular to assess its role in comparison with or as an adjunct to invasive intervention. A systematic literature search revealed a total 11 randomised studies inclusive of 969 patients. All studies compared SET and intervention with monotherapy. Study results suggest that exercise is a complication-free treatment. Furthermore, it appears to offer significant improvements in patients walk distances with a combination of both SET and intervention offering a superior walking outcome to monotherapy in those requiring invasive measures.
\end{abstract}

\section{Introduction}

Peripheral arterial disease (PAD) affects $12-16 \%$ of the population over the age of 60 years with intermittent claudication (IC), its primary symptom, proving detrimental to patient quality of life [1-4]. Typically PAD follows a stable course with management confined to conservative measures; however one in ten PAD patients will develop critical limb ischaemia (CLI) with all-cause mortality in the CLI cohort rising to 50\% at 5 years [5-7]. This reduction in life expectancy is due largely to concomitant cardiovascular disease $[8,9]$.

Therefore, treatment goals should focus not only on the alteration of disease progression and symptomatic relief but also on the improvement of patient long-term survival [10]. Approaches include the modification of risk factors through optimum medical therapy (OMT) and supervised exercise therapy (SET) with endovascular (EVR) and open surgical revascularization reserved for those failing conservative measures. Further novel therapies including kinesitherapy and electrotherapeutic procedures have also been proposed [11]. While endovascular treatment offers a minimally invasive revascularization option for many patients data supporting its ability to improve long-term survival is lacking. Regular exercise, on the other hand, is associated with a 50\% reduction in cardiovascular mortality [12]. Supervised exercise training consists of a prescribed, evidence based exercise program which is performed under the direct observation of a trained practitioner. It is now well established as an initial noninvasive option in all PAD patients with robust supporting data [13-15].

Rationale for Review. Despite its intuitive benefits a wide variation in the use and availability of SET exists and it remains a greatly underutilized resource due to limited patient access [16]. The BASIL study highlights the deficiencies in the current medical optimization with few participants utilizing 
TABLE 1: Treatment groups and disease level.

\begin{tabular}{|c|c|c|c|c|c|c|c|c|c|c|}
\hline \multirow[b]{2}{*}{ Study } & \multirow{2}{*}{$\begin{array}{l}\text { Patient } \\
\text { number }\end{array}$} & \multicolumn{6}{|c|}{ Intervention } & \multicolumn{3}{|c|}{ Disease level } \\
\hline & & EVR & SET & $\begin{array}{c}\text { EVR + } \\
\text { SET }\end{array}$ & INV & Surgery & $\begin{array}{c}\text { Surgery } \\
+ \text { SET }\end{array}$ & Fem-pop & Aortoiliac & Multilevel \\
\hline Lundgren et al. [30] & 75 & & 25 & & & 25 & 25 & & Not recorded & \\
\hline Creasy et al./Perkins et al. $[19,20]$ & 56 & 30 & 26 & & & & & 28 & 25 & 0 \\
\hline Gelin et al. [29] & 164 & & 88 & & 66 & & & & Not recorded & \\
\hline Hobbs et al. [21] & 23 & 9 & 7 & & & & & 23 & 0 & 0 \\
\hline Badger et al. [31] & 14 & & 6 & & & & 8 & 14 & 0 & 0 \\
\hline Greenhalgh et al. [26] & 127 & & 60 & 67 & & & & 93 & 94 & \\
\hline Kruidenier et al. [27] & 70 & 35 & & 35 & & & & 5 & 60 & 5 \\
\hline Mazari et al. [25] & 178 & 60 & 60 & 58 & & & & & Not recorded & \\
\hline Spronk et al./Fakhry et al. [22-24] & 151 & 76 & 75 & & & & & 44 & 106 & - \\
\hline Murphy et al. [18, 32] & 111 & 46 & 43 & & & & & 0 & 111 & 0 \\
\hline Bø et al. [28] & 50 & 21 & & 29 & & & & 25 & 25 & 0 \\
\hline
\end{tabular}

EVR: endovascular revascularization; SET: supervised exercise therapy; INV: invasive management; Fem-pop: femoropopliteal.

clinically proven best medical therapy $[7,17]$. The issue is further clouded by conflicting literature as to the optimal nonsurgical management of these patients [18-32]. Thus while its use is strongly supported by current literature it appears that SET is underutilized in mild-to-moderate PAD while its use in more advanced disease requires further clarification. With this likely in mind the Institute of Medicine has prioritized research into the comparative efficacy of the different treatment modalities for PAD [33]. Furthermore, the role of SET as an adjunct to or substitute for intervention remains unclear.

The aim of this review was to compare the use of supervised exercise therapy to invasive measures in the management of symptomatic peripheral arterial disease thus clarifying an exact role for SET in the management of this patient cohort.

\section{Methods}

2.1. Study Eligibility. All randomised controlled trials (RT) assessing exercise in conjunction with or in comparison to an endovascular or open intervention in the management of peripheral arterial disease were included for review (Table 1). All observational and review data were excluded from the results. Relevant papers were searched and evaluated independently by two assessors. Outcomes were tabulated where figures were included.

2.2. Literature Search. The online medical literature database PUBMED was systematically searched. All studies and relevant reviews were manually cross-referenced to identify any outstanding articles.

PubMed was last searched on September 18, 2015 (Figure 1). The database was comprehensively searched without date or language restriction using the following search strategy.

[[[][[][peripheral arterial disease] OR peripheral vascular disease] OR claudication] AND angioplasty] OR revascularization] OR endovascular] OR open surgery] OR bypass] AND exercise. A total of 8544 studies were identified. After the filter for randomised controlled trials was applied 820 studies were identified. Relevant full articles were reviewed by two reviewers [TA, PN].

\section{Results}

A total of 15 papers (Table 1 ) report outcomes of 11 RT. These trials include a total of 969 patients and all directly compare supervised exercise with various invasive interventions. Maximum walking distance (MWD), intermittent claudication distance (ICD), and ankle brachial pressure index (ABPI) measurement form the cornerstones of vascular assessment in each study.

3.1. Quality Assessment of Assessed Data. The risk of bias in each included study is summarised in Table 2. Few papers reported any participant heterogeneity with regard to baseline function, comorbidities, and smoking status. Risk assessment was performed with guidance from the Cochrane Handbook for Systematic Reviews of Interventions [34].

\subsection{Supervised Exercise versus Endovascular Intervention.} Five trials including 519 patients directly compare the outcomes of EVR and SET in the management of peripheral arterial disease (Table 3).

At six months Murphy et al. reported significant improvements in maximum walk times in those undergoing SET compared to those in the EVR group [18]. However, at 18-month follow-up this benefit was lost with no significant difference in walk times identified [32]. ABPI were consistently higher in the EVR group. Creasy and Perkins noted significant improvements in the functionality of both groups [19, 20]. Again, no significant change in ABPI was noted in any SET cohort. Improvement in mobility was most significant when the disease affected the superficial femoral artery. Hobbs et al. only noted significant improvements in walk distances in those receiving EVR [21]. No improvement was seen with SET alone. Spronk et al. reported a 1-week clinical success rate of $88 \%$ following EVR decreasing to $68 \%$ at 12 months while the SET group had an early success rate of 
TABLE 2: Assessment of bias

\begin{tabular}{|c|c|c|c|}
\hline \multirow{6}{*}{ Lundgren et al. [30] } & Random sequence generation & Randomised but not described & Unclear risk of bias \\
\hline & Allocation concealment & Randomised but not described & Unclear risk of bias \\
\hline & Blinding of participants and personnel & Blinding not possible & Not assessed \\
\hline & Incomplete outcome data & Follow-up data in each group incomplete & Moderate risk of bias \\
\hline & Selective reporting & Clear outcomes & Low risk of bias \\
\hline & Other sources of bias & None & Low risk of bias \\
\hline \multirow{6}{*}{$\begin{array}{l}\text { Creasy et al./Perkins et al. } \\
{[19,20]}\end{array}$} & Random sequence generation & Randomised but not described & Unclear risk of bias \\
\hline & Allocation concealment & Randomised but not described & Unclear risk of bias \\
\hline & Blinding of participants and personnel & Blinding not possible & Not assessed \\
\hline & Incomplete outcome data & No loss to follow-up reported & Low risk of bias \\
\hline & Selective reporting & Clear outcomes & Low risk of bias \\
\hline & Other sources of bias & None & Low risk of bias \\
\hline \multirow{6}{*}{ Gelin et al. [29] } & Random sequence generation & $\begin{array}{l}\text { Randomised via computer based } \\
\text { algorithm }\end{array}$ & Low risk of bias \\
\hline & Allocation concealment & Randomised via computer based system & Low risk of bias \\
\hline & Blinding of participants and personnel & Blinding not possible & Not assessed \\
\hline & Incomplete outcome data & Some loss to follow-up & Moderate risk of bias \\
\hline & Selective reporting & Clear outcomes & Low risk of bias \\
\hline & Other sources of bias & None & Low risk of bias \\
\hline \multirow{6}{*}{ Hobbs et al. [21] } & Random sequence generation & Randomised with $2 \times 2$ factorial design & Low risk of bias \\
\hline & Allocation concealment & Computer generated randomisation & Low risk of bias \\
\hline & Blinding of participants and personnel & Blinding not possible & Not assessed \\
\hline & Incomplete outcome data & Four withdrawals & Low risk of bias \\
\hline & Selective reporting & Clear outcomes & Low risk of bias \\
\hline & Other sources of bias & None & Low risk of bias \\
\hline \multirow{6}{*}{ Badger et al. [31] } & Random sequence generation & Randomised but not described & Unclear risk of bias \\
\hline & Allocation concealment & Randomised but not described & Unclear risk of bias \\
\hline & Blinding of participants and personnel & Blinding not possible & Not assessed \\
\hline & Incomplete outcome data & All patients lost to 6-month follow-up & High risk of bias \\
\hline & Selective reporting & Clear outcomes & Low risk of bias \\
\hline & Other sources of bias & None & Low risk of bias \\
\hline \multirow{6}{*}{ Greenhalgh et al. [26] } & Random sequence generation & $\begin{array}{l}\text { Detailed description of Stata generated } \\
\text { randomisation }\end{array}$ & Low risk of bias \\
\hline & Allocation concealment & Computer generated randomisation & Low risk of bias \\
\hline & Blinding of participants and personnel & Blinding not possible & Not assessed \\
\hline & Incomplete outcome data & Moderate loss to follow-up & Moderate risk of bias \\
\hline & Selective reporting & Clear outcomes & Low risk of bias \\
\hline & Other sources of bias & None & Low risk of bias \\
\hline \multirow{6}{*}{ Kruidenier et al. [27] } & Random sequence generation & Computer generated block randomisation & Low risk of bias \\
\hline & Allocation concealment & Computer generated block randomisation & Low risk of bias \\
\hline & Blinding of participants and personnel & No blinding & High risk of bias \\
\hline & Incomplete outcome data & Moderate losses to follow-up & Moderate risk of bias \\
\hline & Selective reporting & Clear outcomes & Low risk of bias \\
\hline & Other sources of bias & None & Low risk of bias \\
\hline \multirow{6}{*}{ Mazari et al. [25] } & Random sequence generation & Sealed envelope used to randomise & Low risk of bias \\
\hline & Allocation concealment & Sealed envelope used to randomise & Low risk of bias \\
\hline & Blinding of participants and personnel & Blinding not described & Unclear risk of bias \\
\hline & Incomplete outcome data & Moderate loss to follow-up & Moderate risk of bias \\
\hline & Selective reporting & Clear outcomes & Low risk of bias \\
\hline & Other sources of bias & None & Low risk of bias \\
\hline
\end{tabular}


TABLE 2: Continued.

\begin{tabular}{|c|c|c|c|}
\hline \multirow{6}{*}{$\begin{array}{l}\text { Spronk et al./Fakhry et al. } \\
{[22-24]}\end{array}$} & Random sequence generation & Computer generated block randomisation & Low risk of bias \\
\hline & Allocation concealment & Computer generated block randomisation & Low risk of bias \\
\hline & Blinding of participants and personnel & Blinding not possible & Not assessed \\
\hline & Incomplete outcome data & $\begin{array}{l}\text { Prolonged study with some loss to } \\
\text { follow-up }\end{array}$ & Moderate risk of bias \\
\hline & Selective reporting & Clear outcomes & Low risk of bias \\
\hline & Other sources of bias & None & Low risk of bias \\
\hline \multirow{6}{*}{ Murphy et al. $[18,32]$} & Random sequence generation & Web based randomisation & Low risk of bias \\
\hline & Allocation concealment & Web based randomisation & Low risk of bias \\
\hline & Blinding of participants and personnel & Observers blinded & Low risk of bias \\
\hline & Incomplete outcome data & $\begin{array}{l}\text { Prolonged study with some loss to } \\
\text { follow-up }\end{array}$ & Moderate risk of bias \\
\hline & Selective reporting & Clear outcomes & Low risk of bias \\
\hline & Other sources of bias & None & Low risk of bias \\
\hline \multirow{6}{*}{ Bø et al. [28] } & Random sequence generation & Computer based randomisation & Low risk of bias \\
\hline & Allocation concealment & Computer based randomisation & Low risk of bias \\
\hline & Blinding of participants and personnel & Observers blinded & Low risk of bias \\
\hline & Incomplete outcome data & No loss to follow-up & Low risk of bias \\
\hline & Selective reporting & Clear outcomes & Low risk of bias \\
\hline & Other sources of bias & None & Low risk of bias \\
\hline
\end{tabular}

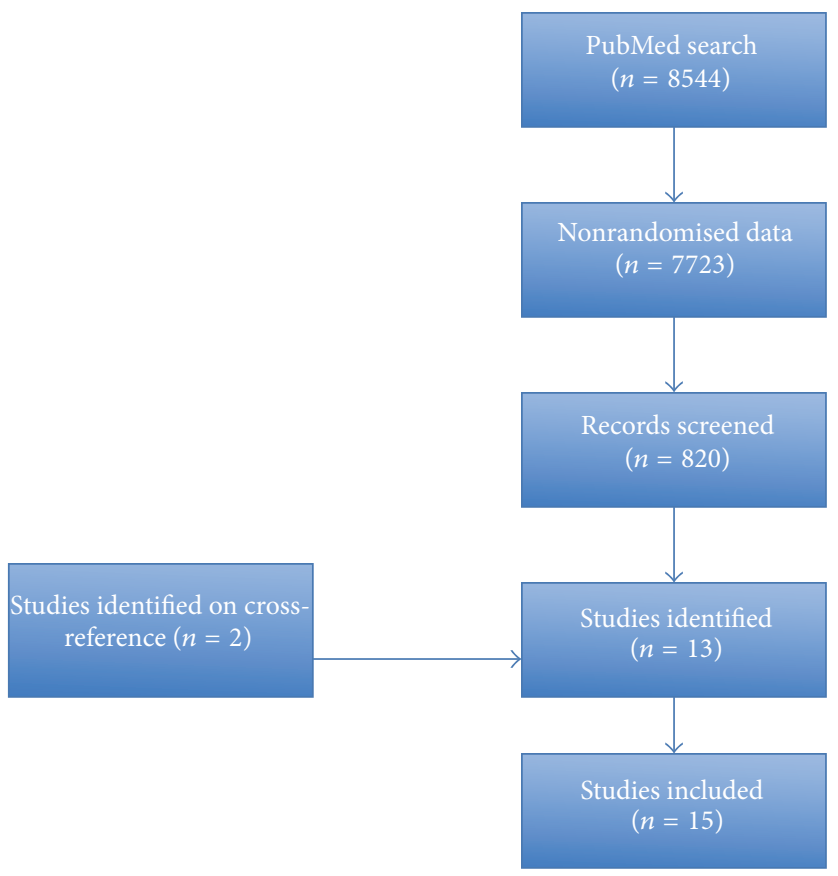

Figure 1: Flow diagram depicting study identification.

$16 \%$ increasing to $65 \%$ by 12 months [22, 23]. Clinical success was defined as an improvement in at least one category in the Rutherford scale posttreatment. Long-term outcomes (7 years) from this RT show maintenance of the functional gains achieved at 1 year with no variance in amputation rate 10 between groups [24]. Finally, Mazari et al. identified significant functional improvements in both groups at one year; however no statistically significant difference was identified between cohorts [25]. Again, only EVR was associated with improved ABPI measurements.
3.3. Supervised Exercise Plus Invasive Measures (Open Surgery or EVR) versus Monotherapy. In total six studies inclusive of 514 patients compare the merits of combination therapy consisting of invasive intervention and SET and single intervention alone (Table 4). Two included studies examine the benefits of a combination of open arterial surgical reconstruction and SET [30, 31] while four articles assess dual therapy including both EVR and SET [25-28].

The earliest work in this area by Lundgren et al. compares open arterial reconstruction and SET with open surgery 
TABLE 3: Supervised exercise versus endovascular revascularization.

\begin{tabular}{|c|c|c|c|c|c|c|c|c|c|c|}
\hline \multirow{2}{*}{ Study } & \multirow{2}{*}{ Follow-up } & \multicolumn{2}{|c|}{ ABPI } & \multirow{2}{*}{$P$} & \multicolumn{2}{|c|}{ MWD } & \multirow{2}{*}{$P$} & \multicolumn{2}{|c|}{ ICD } & \multirow{2}{*}{$P$} \\
\hline & & Baseline & Endpoint & & Baseline & Endpoint & & Baseline & Endpoint & \\
\hline \multicolumn{11}{|c|}{ Hobbs et al. [21] } \\
\hline EVR & \multirow{2}{*}{6 months } & 0.69 & 0.93 & $0.013^{\dagger}$ & 185 & 698 & $0.008^{\dagger}$ & 84 & 698 & $0.011^{\dagger}$ \\
\hline SET & & 0.66 & 0.70 & $0.46^{\dagger}$ & 111 & 124 & $0.35^{\dagger}$ & 59 & 92 & $0.074^{\dagger}$ \\
\hline \multicolumn{11}{|c|}{$\begin{array}{l}\text { Mazari et al. } \\
\text { [25] }\end{array}$} \\
\hline EVR & \multirow{2}{*}{12 months } & 0.71 & 0.90 & & 77 & 146 & & 31 & 75 & \\
\hline SET & & 0.72 & 0.84 & 0.093 & 83 & 215 & 0.2 & 42 & 97 & 0.48 \\
\hline \multicolumn{11}{|c|}{$\begin{array}{l}\text { Spronk et } \\
\text { al./Fakhry et al. } \\
{[22-24]}\end{array}$} \\
\hline EVR & \multirow{2}{*}{7 years } & 0.63 & 0.84 & & 174 & 1248 & & 82 & 1022 & \\
\hline SET & & 0.62 & 0.82 & 0.8 & 186 & 1168 & 0.48 & 104 & 804 & 0.15 \\
\hline \multicolumn{11}{|c|}{$\begin{array}{l}\text { Murphy et al. } \\
{[18,32]}\end{array}$} \\
\hline EVR & \multirow{2}{*}{18 months } & 0.7 & 0.7 & & $5.2 \mathrm{~min}$ & $8.4 \mathrm{~min}$ & & $1.8 \mathrm{~min}$ & $4.8 \mathrm{~min}$ & \\
\hline SET & & 0.6 & 0.6 & $<0.001$ & $5.6 \mathrm{~min}$ & $10.6 \mathrm{~min}$ & 0.16 & $1.8 \mathrm{~min}$ & $5.1 \mathrm{~min}$ & 0.77 \\
\hline
\end{tabular}

ABPI: ankle brachial pressure index; MWD: maximal walk distance; ICD: intermittent claudication distance; EVR: endovascular revascularization; SET: supervised exercise therapy. Distances in metres unless otherwise stated. min represents time in minutes, claudication onset time/maximal walk time. $P$ value represents statistical comparison of interventions except in Hobbs et al. [21] where $\dagger$ represents change in measurement over study period.

TABLE 4: Supervised exercise plus invasive measures (open surgery or EVR) versus monotherapy.

\begin{tabular}{|c|c|c|c|c|c|c|c|c|c|c|}
\hline \multirow{2}{*}{ Study } & \multirow{2}{*}{ Follow-up } & \multicolumn{2}{|c|}{ ABPI } & \multirow{2}{*}{$P$} & \multicolumn{2}{|c|}{ MWD } & \multirow{2}{*}{$P$} & \multicolumn{2}{|c|}{ ICD } & \multirow{2}{*}{$P$} \\
\hline & & Baseline & Endpoint & & Baseline & Endpoint & & Baseline & Endpoint & \\
\hline \multicolumn{11}{|c|}{ Lundgren et al. [30] } \\
\hline Surgery & \multirow{3}{*}{13 months } & 0.55 & - & & 209 & 570 & & 85 & 405 & \\
\hline SET & & 0.59 & - & & 180 & 654 & & 70 & 559 & \\
\hline Surgery + SET & & 0.59 & - & $<0.001$ & 183 & 459 & 0.05 & 67 & 187 & 0.006 \\
\hline \multicolumn{11}{|l|}{ Badger et al. [31] } \\
\hline Surgery & \multirow{2}{*}{6 months } & - & - & & - & - & & - & - & - \\
\hline Surgery + SET & & - & - & $0.02^{*}$ & - & - & $0.001^{*}$ & - & - & - \\
\hline \multicolumn{11}{|c|}{$\begin{array}{l}\text { Greenhalgh et al. [26] } \\
\text { (Aortoiliac) }\end{array}$} \\
\hline SET & \multirow{2}{*}{24 months } & 0.66 & 0.74 & & 126 & 168 & & - & - & - \\
\hline $\mathrm{EVR}+\mathrm{SET}$ & & 0.68 & 0.90 & 0.02 & 114 & 354 & 0.05 & - & - & - \\
\hline \multicolumn{11}{|c|}{$\begin{array}{l}\text { Greenhalgh et al. [26] } \\
\text { (Femoropopliteal) }\end{array}$} \\
\hline SET & \multirow{2}{*}{24 months } & 0.69 & 0.72 & & 126 & 155 & & - & - & - \\
\hline $\mathrm{EVR}+\mathrm{SET}$ & & 0.66 & 0.83 & 0.01 & 133 & 245 & 0.4 & - & - & - \\
\hline \multicolumn{11}{|c|}{ Kruidenier et al. [27] } \\
\hline EVR & \multirow{2}{*}{6 months } & 0.71 & 0.93 & & 282 & 685 & & 343 & 547 & \\
\hline $\mathrm{EVR}+\mathrm{SET}$ & & 0.69 & 0.88 & 0.755 & 186 & 956 & 0.001 & 293 & 842 & 0.001 \\
\hline \multicolumn{11}{|l|}{ Mazari et al. [25] } \\
\hline EVR & \multirow{3}{*}{12 months } & 0.71 & 0.9 & & 77 & 146 & & 31 & 75 & \\
\hline SET & & 0.72 & 0.84 & & 83 & 215 & & 42 & 97 & \\
\hline $\mathrm{EVR}+\mathrm{SET}$ & & 0.64 & 0.92 & 0.93 & 85 & 187 & 0.259 & 43 & 99 & 0.484 \\
\hline \multicolumn{11}{|l|}{ Bø et al. [28] } \\
\hline EVR & \multirow{2}{*}{3 months } & - & - & & 213 & 427 & & 94 & 267 & \\
\hline $\mathrm{EVR}+\mathrm{SET}$ & & - & - & $<0.001^{*}$ & 385 & 584 & NS & 101 & 456 & NS \\
\hline
\end{tabular}

ABPI: ankle brachial pressure index; MWD: maximal walk distance; ICD: intermittent claudication distance; EVR: endovascular revascularization; SET: supervised exercise therapy. Distances in metres unless otherwise stated. $*$ represents significant improvement favouring combined treatment. $P$ value represents statistical comparison of interventions. - represents nonreported figures. 
TABLE 5: Invasive (EVR or surgery) management versus supervised exercise.

\begin{tabular}{|c|c|c|c|c|c|c|c|c|c|c|}
\hline \multirow{2}{*}{ Study } & \multirow{2}{*}{ Follow-up } & \multicolumn{2}{|c|}{ ABPI } & \multirow{2}{*}{$P$} & \multicolumn{2}{|c|}{ MWD } & \multirow{2}{*}{$P$} & \multicolumn{2}{|c|}{ ICD } & \multirow{2}{*}{$P$} \\
\hline & & Baseline & Endpoint & & Baseline & Endpoint & & Baseline & Endpoint & \\
\hline \multicolumn{11}{|c|}{ Gelin et al. [29] } \\
\hline INV & \multirow{2}{*}{12 months } & 0.55 & 0.71 & $<0.01^{\dagger}$ & 274 & 344 & $<0.01^{\dagger}$ & - & - & - \\
\hline SET & & 0.56 & 0.54 & - & 258 & 247 & - & - & - & - \\
\hline
\end{tabular}

ABPI: ankle brachial pressure index; MWD: maximal walk distance; ICD: intermittent claudication distance; EVR: endovascular revascularization; SET: supervised exercise therapy. Distances in metres unless otherwise stated. $P$ value represents statistical comparison of interventions. $\dagger$ represents change in measurement over study period.

alone [30]. This study identified improvements in walk distances in both groups; however, those undergoing combination therapy experienced significantly improved walking performance at 13 months. Similarly, Badger et al. identified significant improvements in MWD in patients undergoing peripheral arterial bypass in conjunction with SET compared to those undergoing bypass in isolation [31].

More recently, studies have focused on the use of SET as an adjunct to EVR. Mazari et al. identified that a combined treatment group achieved the greatest (but not statistically significant) improvement in MWD and ICD with a lower incidence of reintervention compared to the monotherapy groups [25]. This benefit was further supported by randomised data from Greenhalgh et al. [26-28]. At six months Kruidenier et al. identified significantly lengthened walk distances when SET was used as an adjunct to EVR. Furthermore, both Greenhalgh and Bø et al. examined patients with both aortoiliac and infrainguinal disease in separate trial limbs. While Greenhalgh compared combination therapy with SET alone Bø et al. contrasted dual therapy with EVR alone. Both studies identified improvements in walk distances in both trial limbs for patients undergoing combination therapy versus monotherapy; however only Greenhalgh identified significantly better ABPI in the dual intervention group.

\subsection{Invasive (EVR or Surgery) Management versus Supervised} Exercise. Gelin alone compared invasive intervention (either EVR or open surgery based on preoperative angiography) and supervised exercise [29] (Table 5). At one year only those randomised to invasive measures experienced any improvement in walk distance or lower limb arterial pressures.

3.5. Open Surgery versus Supervised Exercise. Lundgren et al. included a comparison of open revascularisation and SET in the previously discussed RT (Table 4) [30]. At 13 months those undergoing open surgery had better functional performance than those undergoing SET alone. In addition, those undergoing surgery experienced a significantly higher ABPI to those in the exercise group.

\section{Discussion}

Peripheral arterial disease is a widespread phenomenon in the elderly population [35]. The optimum management of claudication continues to raise debate. Exercise is a straightforward and effective conservative treatment option. Meta-analysis has shown a $122 \%$ increase in walking distances in patients undergoing exercise therapy [15]. This has been reinforced by the more recent Cochrane review examining exercise for IC [36]. However, despite its success intensive programs continue to be associated with high dropout rates [37].

The exact physiological mechanism by which exercise improves performance is incompletely understood. Multiple physiological adaptions have been proposed as contributing factors. Arterial collateralization has the potential to improve peripheral blood flow in the ischaemic limb with the exercised muscle displaying increased levels of the proangiogenic vascular endothelial growth factor [38]. However, improved functional performance in the trained limb is not reflective of improved ABPI measurements as seen in endovascular revascularization [18, 39]. Increased arterial shear stress in exercise is associated with nitric oxide (NO) release, a powerful vasoactive agent [40]. This endothelial-mediated response is impaired in patients with PAD [41]. The concept of "hemorheologic fitness" suggests that a proven reduction in blood viscosity in the trained individual may result in improved peripheral metabolic efficiency [42]. In addition, gait proficiency, reversal of acquired metabolic myopathies, and modified inflammatory responses all have the potential to improve exercise related function [43-46].

This review suggests a number of roles for supervised exercise therapy in the symptomatic PAD patient (Figure 2). Significantly, no study reported exercise related complications.

(i) Direct comparison of SET and endovascular measures revealed similar functional outcomes for both interventions in the medium term across a number of studies with long-term data from one study identifying comparable limb salvage between groups. However, only EVR alone resulted in significant improvements in lower limb perfusion as measured by ABPI. These data have significant applicability to symptomatic patients whose comorbidities allow them to exercise to an adequate level for SET. Supervised exercise offers this group an acceptable, effective initial step in those capable and motivated to take part.

(ii) In those requiring intervention a combination of surgical intervention and SET offered superior outcomes to monotherapy across multiple studies. Two trials assessed open surgery with adjunctive SET with both suggesting significant benefits in walk distances 


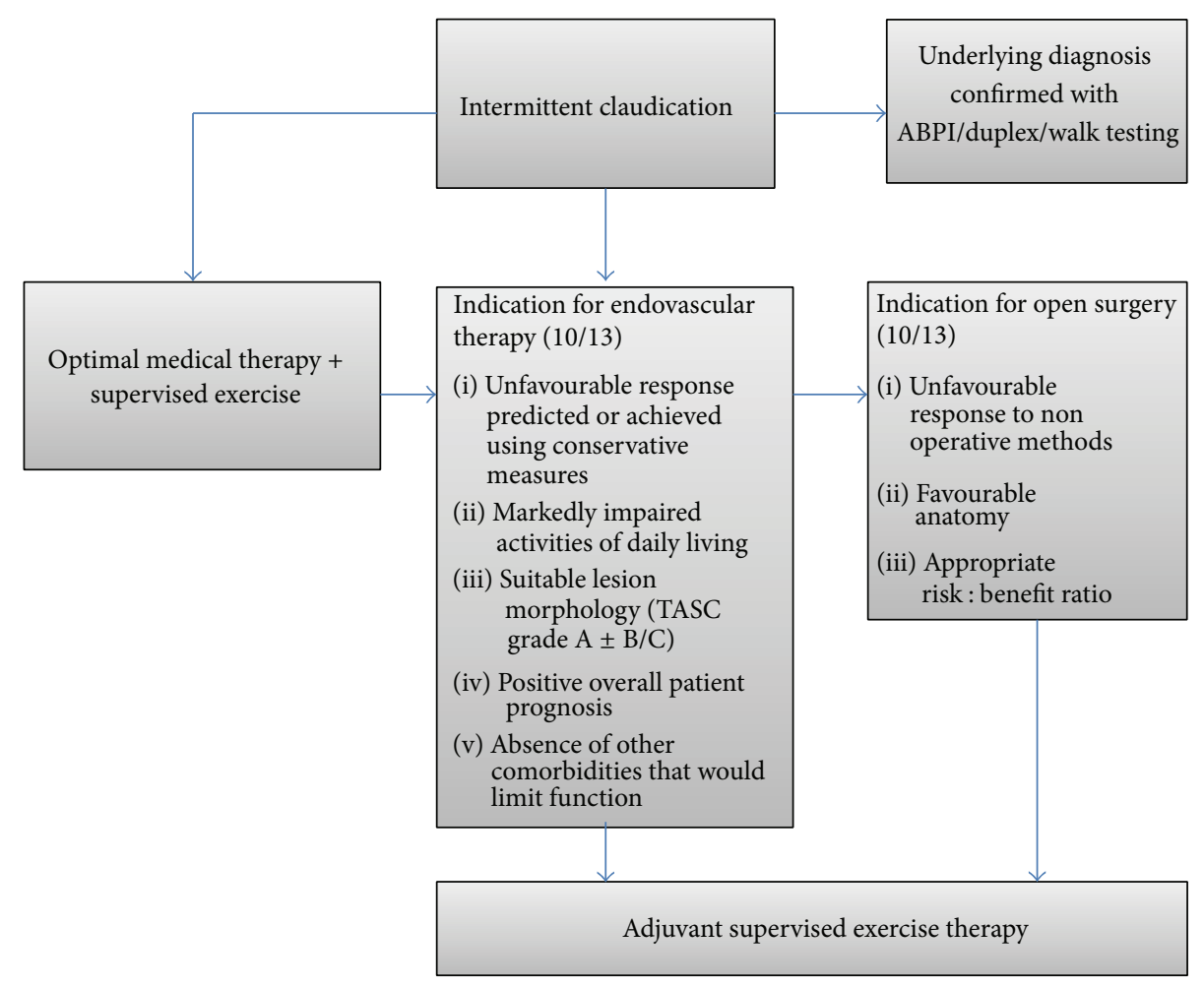

FIGURE 2: Management of intermittent claudication incorporating supervised exercise therapy.

in the dual therapy group. Similarly, all four trials assessing endovascular intervention in conjunction with SET compared to monotherapy found greater improvements in the walking distances of those undergoing dual therapy. These data would strongly support the use of SET as an adjunct to any operative intervention in the management of symptomatic PAD.

(iii) Only one study directly compared open surgery and SET. This strongly supported the surgical approach in terms of both function and perfusion outcomes.

Multiple exercise modalities including treadmill walking, resistance training, and upper limb ergometry have proven benefit in PAD [12, 36, 47, 48]. Unfortunately, the success of SET is reliant on excellent compliance and availability. Supervised exercise training has been shown to consistently result in improved functionality with superior outcomes to a "go home and walk" approach [49-51]. Compliance can further be augmented by regular exercise reminders, patient specific exercise prescriptions, target setting, and the incorporation of exercise into daily activities [52]. Gains in walk time and claudication onset occur rapidly over the initial 2 months and can be maintained with good compliance [53]. An exercise prescription of at least two 30 -minute sessions per week offers optimal walking outcomes [54].

In those failing or unable to partake in an initial exercise program endovascular revascularization offers low rates of periprocedural morbidity and mortality with excellent initial success rates [55-57]. However the two main shortcomings of endovascular intervention include long-term durability and overall poor survival rates associated with concomitant cardiovascular disease. Supervised exercise addresses some of these concerns. By stimulating collateralization and the release of vasodilators such as nitric oxide exercise therapy should confer superior functional results in the longer-term. This is supported by a number of studies $[58,59]$. Thus, for this patient group SET offers not only functional improvement but also significant benefits to the cardiovascular health of these patients [12].

4.1. Strengths and Limitations. This review is strengthened by the fact that all incorporated data are from well-designed randomised controlled studies. Follow-up ranged from 6 months to 12 years. Cumulatively these trials incorporate a significant number of claudicants. Individually papers provide strong data that exercise results in significant gains in walk distance. In addition, a number of studies have found that a combination of SET and intervention provides a superior outcome to monotherapy.

This review is however subject to a number of limitations. Firstly, while all patients had significant arterial symptoms a degree of heterogeneity exists in this cohort. Each included study describes slight variations in the level of disease, the symptomatic presentation, and the intervention received rendering definitive conclusion difficult. Secondly, many studies included small numbers of participants with a number reporting moderate losses to follow-up. Finally, due to the heterogeneity of outcome data meta-analysis was not performed as part of this review. 


\section{Conclusion}

Exercise is an effective, safe, and economical method of treating symptomatic PAD [23]. Some randomised data now suggests that it may be comparable to EVR in the management of symptomatic noncritical ischaemia. Furthermore there is emerging evidence that combining endovascular intervention following by supervised exercise achieves better long-term results than endovascular intervention alone; however greater surgeon access to and awareness of this important adjuvant therapy is essential to maximize therapeutic outcomes.

\section{Conflict of Interests}

The authors declare that there is no conflict of interests regarding the publication of this paper.

\section{References}

[1] M. H. Criqui, A. Fronek, E. Barrett-Connor, M. R. Klauber, S. Gabriel, and D. Goodman, "The prevalence of peripheral arterial disease in a defined population," Circulation, vol. 71, no. 3, pp. 510-515, 1985.

[2] M. Schroll and O. Munck, "Estimation of peripheral arteriosclerotic disease by ankle blood pressure measurements in a population study of 60-year-old men and women," Journal of Chronic Diseases, vol. 34, no. 6, pp. 261-269, 1981.

[3] E. Selvin and T. P. Erlinger, "Prevalence of and risk factors for peripheral arterial disease in the United States: results from the National Health and Nutrition Examination Survey, 19992000," Circulation, vol. 110, no. 6, pp. 738-743, 2004.

[4] I. C. Chetter, P. Dolan, J. I. Spark, D. J. A. Scott, and R. C. Kester, "Correlating clinical indicators of lower-limb ischaemia with quality of life," Cardiovascular Surgery, vol. 5, no. 4, pp. 361-366, 1997.

[5] K. Bloor, "Natural history of arteriosclerosis of the lower extremities-hunterian lecture delivered at the Royal College of Surgeons of England on 22nd April 1960," Annals of the Royal College of Surgeons of England, vol. 28, no. 1, pp. 36-52, 1961.

[6] J. A. Dormandy, L. Heeck, and S. Vig, "The fate of patients with critical leg ischemia," Seminars in Vascular Surgery, vol. 12, no. 2, pp. 142-147, 1999.

[7] A. W. Bradbury, D. J. Adam, J. D. Beard et al., "Bypass versus angioplasty in severe ischaemia of the leg (BASIL): multicentre, randomised controlled trial," The Lancet, vol. 366, no. 9501, pp. 1925-1934, 2005.

[8] M. H. Criqui, R. D. Langer, A. Fronek et al., "Mortality over a period of 10 years in patients with peripheral arterial disease," The New England Journal of Medicine, vol. 326, no. 6, pp. 381386, 1992.

[9] F. G. R. Fowkes, "Epidemiology of atherosclerotic arterial disease in the lower limbs," European Journal of Vascular Surgery, vol. 2, no. 5, pp. 283-291, 1988.

[10] J. A. Dormandy and R. B. Rutherford, "Management of peripheral arterial disease (PAD). TASC Working Group. TransAtlantic Inter-Society Consensus (TASC)," Journal of Vascular Surgery, vol. 31, no. 1, part 2, pp. S1-S296, 2000.
[11] M. D. Markovic, D. M. Markovic, M. V. Dragas et al., "The role of kinesitherapy and electrotherapeutic procedures in nonoperative management of patients with intermittent claudications," Vascular, 2015.

[12] K. E. Powell and M. Pratt, "Physical activity and health," British Medical Journal, vol. 313, no. 7050, pp. 126-127, 1996.

[13] A. T. Hirsch, Z. J. Haskal, N. R. Hertzer et al., "ACC/AHA 2005 guidelines for the management of patients with peripheral arterial disease (lower extremity, renal, mesenteric, and abdominal aortic): executive summary a collaborative report from the American Association for Vascular Surgery/Society for Vascular Surgery, Society for Cardiovascular Angiography and Interventions, Society for Vascular Medicine and Biology, Society of Interventional Radiology, and the ACC/AHA Task Force on practice guidelines (writing committee to develop guidelines for the management of patients with peripheral arterial disease): endorsed by the American Association of Cardiovascular and Pulmonary Rehabilitation; National Heart, Lung, and Blood Institute; Society for Vascular Nursing; TransAtlantic InterSociety Consensus; and Vascular Disease Foundation," Journal of the American College of Cardiology, vol. 47, no. 6, pp. 12391312, 2006.

[14] L. Norgren, W. R. Hiatt, and J. A. Dormandy, "Inter-society consensus for the management of peripheral arterial disease (TASC II)," European Journal of Vascular and Endovascular Surgery, vol. 33, supplement 1, pp. S1-S75, 2007.

[15] A. W. Gardner and E. T. Poehlman, "Exercise rehabilitation programs for the treatment of claudication pain. A metaanalysis," The Journal of the American Medical Association, vol. 274, no. 12, pp. 975-980, 1995.

[16] A. H. Stewart and P. M. Lamont, "Exercise for intermittent claudication. Supervised programmes should be universally available," The British Medical Journal, vol. 323, no. 7315, pp. 703-704, 2001.

[17] P. Burns, S. Gough, and A. W. Bradbury, "Management of peripheral arterial disease in primary care," British Medical Journal, vol. 326, no. 7389, pp. 584-588, 2003.

[18] T. P. Murphy, D. E. Cutlip, J. G. Regensteiner et al., "Supervised exercise versus primary stenting for claudication resulting from aortoiliac peripheral artery disease: six-month outcomes from the claudication: exercise versus endoluminal revascularization (CLEVER) study," Circulation, vol. 125, no. 1, pp. 130-139, 2012.

[19] T. S. Creasy, P. J. McMillan, E. W. L. Fletcher, J. Collin, and P. J. Morris, "Is percutaneous transluminal angioplasty better than exercise for claudication? Preliminary results from a prospective randomised trial," European Journal of Vascular Surgery, vol. 4, no. 2, pp. 135-140, 1990.

[20] J. M. T. Perkins, J. Collin, T. S. Creasy, E. W. L. Fletcher, and P. J. Morris, "Exercise training versus angioplasty for stable claudication. Long and medium term results of a prospective, randomised trial," European Journal of Vascular and Endovascular Surgery, vol. 11, no. 4, pp. 409-413, 1996.

[21] S. D. Hobbs, T. Marshall, C. Fegan, D. J. Adam, and A. W. Bradbury, "The constitutive procoagulant and hypofibrinolytic state in patients with intermittent claudication due to infrainguinal disease significantly improves with percutaneous transluminal balloon angioplasty," Journal of Vascular Surgery, vol. 43, no. 1, pp. 40-46, 2006.

[22] S. Spronk, J. L. Bosch, P. T. den Hoed, H. F. Veen, P. M. T. Pattynama, and M. G. M. Hunink, "Intermittent claudication: clinical effectiveness of endovascular revascularization versus supervised hospital-based exercise training-randomized controlled trial," Radiology, vol. 250, no. 2, pp. 586-595, 2009. 
[23] S. Spronk, J. L. Bosch, P. T. den Hoed, H. F. Veen, P. M. T. Pattynama, and M. G. M. Hunink, "Cost-effectiveness of endovascular revascularization compared to supervised hospital-based exercise training in patients with intermittent claudication: a randomized controlled trial," Journal of Vascular Surgery, vol. 48, no. 6, pp. 1472-1480, 2008.

[24] F. Fakhry, E. V. Rouwet, P. T. Den Hoed, M. G. M. Hunink, and S. Spronk, "Long-term clinical effectiveness of supervised exercise therapy versus endovascular revascularization for intermittent claudication from a randomized clinical trial," British Journal of Surgery, vol. 100, no. 9, pp. 1164-1171, 2013.

[25] F. A. K. Mazari, J. A. Khan, D. Carradice et al., "Randomized clinical trial of percutaneous transluminal angioplasty, supervised exercise and combined treatment for intermittent claudication due to femoropopliteal arterial disease," British Journal of Surgery, vol. 99, no. 1, pp. 39-48, 2012.

[26] R. M. Greenhalgh, J. J. Belch, L. C. Brown, P. A. Gaines, L. Gao, and J. A. Reise, "The adjuvant benefit of angioplasty in patients with mild to moderate intermittent claudication (MIMIC) managed by supervised exercise, smoking cessation advice and best medical therapy: results from two randomised trials for stenoticfemoropopliteal and aortoiliac arterial disease," European Journal of Vascular and Endovascular Surgery, vol. 36, no. 6, pp. 680-688, 2008.

[27] L. M. Kruidenier, S. P. Nicolaï, E. V. Rouwet, R. J. Peters, M. H. Prins, and J. A. W. Teijink, "Additional supervised exercise therapy after a percutaneous vascular intervention for peripheral arterial disease: a randomized clinical trial," Journal of Vascular and Interventional Radiology, vol. 22, no. 7, pp. 961968, 2011.

[28] E. Bø, J. Hisdal, M. Cvancarova et al., "Twelve-months followup of supervised exercise after percutaneous transluminal angioplasty for intermittent claudication: a randomised clinical trial," International Journal of Environmental Research and Public Health, vol. 10, no. 11, pp. 5998-6014, 2013.

[29] J. Gelin, L. Jivegård, C. Taft et al., "Treatment efficacy of intermittent claudication by surgical intervention, supervised physical exercise training compared to no treatment in unselected randomised patients I: one year results of functional and physiological improvements," European Journal of Vascular and Endovascular Surgery, vol. 22, no. 2, pp. 107-113, 2001.

[30] F. Lundgren, A.-G. Dahllof, K. Lundholm, T. Schersten, and R. Volkmann, "Intermittent claudication-surgical reconstruction or physical training? A prospective randomized trial of treatment efficiency," Annals of Surgery, vol. 209, no. 3, pp. 346-355, 1989.

[31] S. A. Badger, C. V. Soong, M. E. O'Donnell, C. A. G. Boreham, and K. E. McGuigan, "Benefits of a supervised exercise program after lower limb bypass surgery," Vascular and Endovascular Surgery, vol. 41, no. 1, pp. 27-32, 2007.

[32] T. P. Murphy, D. E. Cutlip, J. G. Regensteiner et al., "Supervised exercise, stent revascularization, or medical therapy for claudication due to aortoiliac peripheral artery disease: the CLEVER study," Journal of the American College of Cardiology, vol. 65, no. 10, pp. 999-1009, 2015.

[33] 100 initial topics for comparative effectiveness research, 2009, http://www.iom.edu/Reports/2009/ComparativeEffectivenessResearchPriorities.aspx.

[34] J. A. C. Sterne, M. Egger, and D. Moher, "Addressing reporting biases," in Cochrane Handbook for Systematic Reviews of Interventions Version 5.1.0, J. P. T. Higgins and S. Green, Eds., The Cochrane Collaboration, 2011.
[35] G. C. Leng, O. Papacosta, P. Whincup et al., "Femoral atherosclerosis in an older British population: prevalence and risk factors," Atherosclerosis, vol. 152, no. 1, pp. 167-174, 2000.

[36] L. Watson, B. Ellis, and G. C. Leng, "Exercise for intermittent claudication," The Cochrane Database of Systematic Reviews, no. 4, Article ID CD000990, 2008.

[37] B. L. Bendermacher, E. M. Willigendael, S. P. Nicolaï et al., "Supervised exercise therapy for intermittent claudication in a community-based setting is as effective as clinic-based," Journal of Vascular Surgery, vol. 45, no. 6, pp. 1192-1196, 2007.

[38] T. Gustafsson, A. Puntschart, L. Kaijser, E. Jansson, and C. J. Sundberg, "Exercise-induced expression of angiogenesisrelated transcription and growth factors in human skeletal muscle," American Journal of Physiology: Heart and Circulatory Physiology, vol. 276, no. 2, pp. H679-H685, 1999.

[39] R. Ekroth, A.-G. Dahllöf, B. Gundevall, J. Holm, and T. Scherstén, "Physical training of patients with intermittent claudication: indications, methods, and results," Surgery, vol. 84, no. 5, pp. 640-643, 1978.

[40] M. Noris, M. Morigi, R. Donadelli et al., "Nitric oxide synthesis by cultured endothelial cells is modulated by flow conditions," Circulation Research, vol. 76, no. 4, pp. 536-543, 1995.

[41] A. R. Yataco, M. C. Corretti, A. W. Gardner, C. J. Womack, and L. I. Katzel, "Endothelial reactivity and cardiac risk factors in older patients with peripheral arterial disease," American Journal of Cardiology, vol. 83, no. 5, pp. 754-758, 1999.

[42] E. Ernst, "Influence of regular physical activity on blood rheology," European Heart Journal, vol. 8, pp. 59-62, 1987.

[43] C. J. Womack, D. J. Sieminski, L. I. Katzel, A. Yataco, and A. W. Gardner, "Improved walking economy in patients with peripheral arterial occlusive disease," Medicine \& Science in Sports \& Exercise, vol. 29, no. 10, pp. 1286-1290, 1997.

[44] T. A. Beckitt, J. Day, M. Morgan, and P. M. Lamont, "Calf muscle oxygen saturation and the effects of supervised exercise training for intermittent claudication," Journal of Vascular Surgery, vol. 56, no. 2, pp. 470-475, 2012.

[45] P. V. Tisi, M. Hulse, A. Chulakadabba, P. Gosling, and C. P. Shearman, "Exercise training for intermittent claudication: does it adversely affect biochemical markers of the exercise-induced inflammatory response?" European Journal of Vascular and Endovascular Surgery, vol. 14, no. 5, pp. 344-350, 1997.

[46] K. J. Stewart, W. R. Hiatt, J. G. Regensteiner, and A. T. Hirsch, "Exercise training for claudication," The New England Journal of Medicine, vol. 347, no. 24, pp. 1941-1951, 2002.

[47] M. M. McDermott, P. Ades, J. M. Guralnik et al., “Treadmill exercise and resistance training in patients with peripheral arterial disease with and without intermittent claudication: a randomized controlled trial," The Journal of the American Medical Association, vol. 301, no. 2, pp. 165-174, 2009.

[48] I. Zwierska, R. D. Walker, S. A. Choksy, J. S. Male, A. G. Pockley, and J. M. Saxton, "Upper-vs lower-limb aerobic exercise rehabilitation in patients with symptomatic peripheral arterial disease: a randomized controlled trial," Journal of Vascular Surgery, vol. 42, no. 6, pp. 1122-1130, 2005.

[49] F. A. Frans, S. Bipat, J. A. Reekers, D. A. Legemate, and M. J. W. Koelemay, "Systematic review of exercise training or percutaneous transluminal angioplasty for intermittent claudication," British Journal of Surgery, vol. 99, no. 1, pp. 16-28, 2012.

[50] A. A. Ahimastos, E. P. Pappas, P. G. Buttner, P. J. Walker, B. A. Kingwell, and J. Golledge, "A meta-analysis of the outcome of endovascular and noninvasive therapies in the treatment of 
intermittent claudication," Journal of Vascular Surgery, vol. 54, no. 5, pp. 1511-1521, 2011.

[51] B. L. Bendermacher, E. M. Willigendael, J. A. Teijink, and M. H. Prins, "Supervised exercise therapy versus non-supervised exercise therapy for intermittent claudication," Cochrane Database of Systematic Reviews, no. 2, Article ID CD005263, 2006.

[52] L. Bourke, K. E. Homer, M. A. Thaha et al., "Interventions for promoting habitual exercise in people living with and beyond cancer," The Cochrane Database of Systematic Reviews, vol. 9, Article ID CD010192, 2013.

[53] A. W. Gardner, P. S. Montgomery, and D. E. Parker, "Optimal exercise program length for patients with claudication," Journal of Vascular Surgery, vol. 55, no. 5, pp. 1346-1354, 2012.

[54] S. P. A. Nicolaï, E. J. M. Hendriks, M. H. Prins, and J. A. W. Teijink, "Optimizing supervised exercise therapy for patients with intermittent claudication," Journal of Vascular Surgery, vol. 52, no. 5, pp. 1226-1233, 2010.

[55] V. G. Papavassiliou, S. R. Walker, A. Bolia, G. Fishwick, and N. J. M. London, "Techniques for the endovascular management of complications following lower limb percutaneous transluminal angioplasty," European Journal of Vascular and Endovascular Surgery, vol. 25, no. 2, pp. 125-130, 2003.

[56] V. S. Kashyap, M. L. Pavkov, J. F. Bena et al., "The management of severe aortoiliac occlusive disease: endovascular therapy rivals open reconstruction," Journal of Vascular Surgery, vol. 48, no. 6, pp. 1451.e3-1457.e3, 2008.

[57] G. S. R. Muradin, J. L. Bosch, T. Stijnen, and M. G. M. Hunink, "Balloon dilation and stent implantation for treatment of femoropopliteal arterial disease: meta-analysis," Radiology, vol. 221, no. 1, pp. 137-145, 2001.

[58] M. M. McDermott, T. J. Carroll, M. Kibbe et al., "Proximal superficial femoral artery occlusion, collateral vessels, and walking performance in peripheral artery disease," JACC: Cardiovascular Imaging, vol. 6, no. 6, pp. 687-694, 2013.

[59] J. D. Allen, T. Stabler, A. Kenjale et al., "Plasma nitrite flux predicts exercise performance in peripheral arterial disease after 3 months of exercise training," Free Radical Biology and Medicine, vol. 49, no. 6, pp. 1138-1144, 2010. 


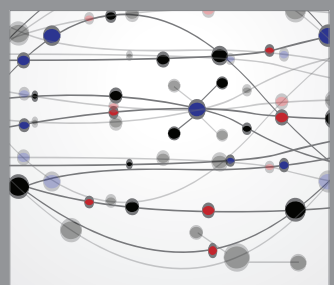

The Scientific World Journal
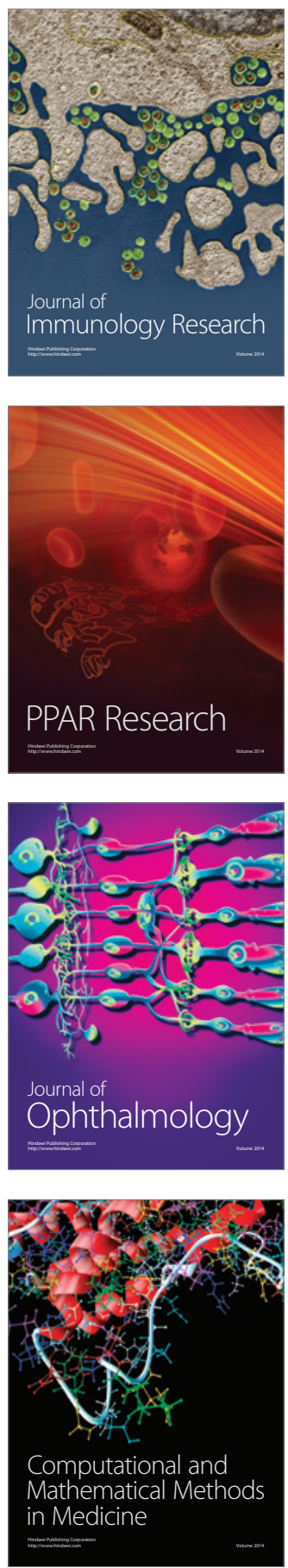

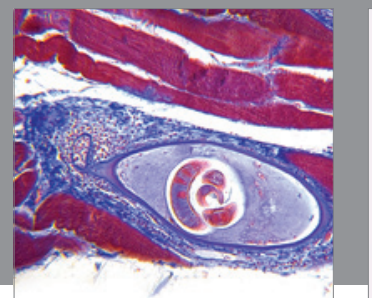

Gastroenterology

Research and Practice
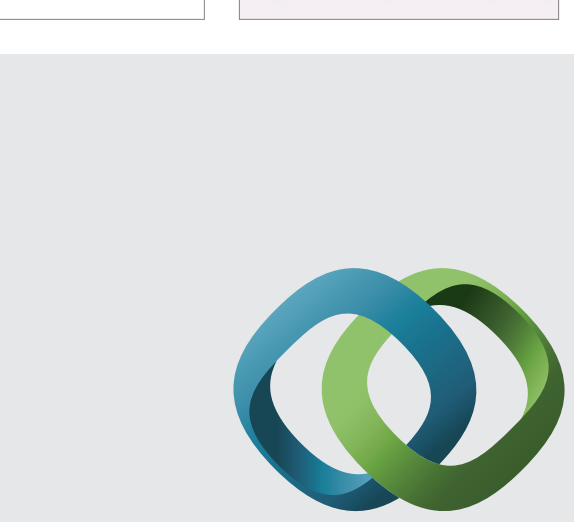

\section{Hindawi}

Submit your manuscripts at

http://www.hindawi.com
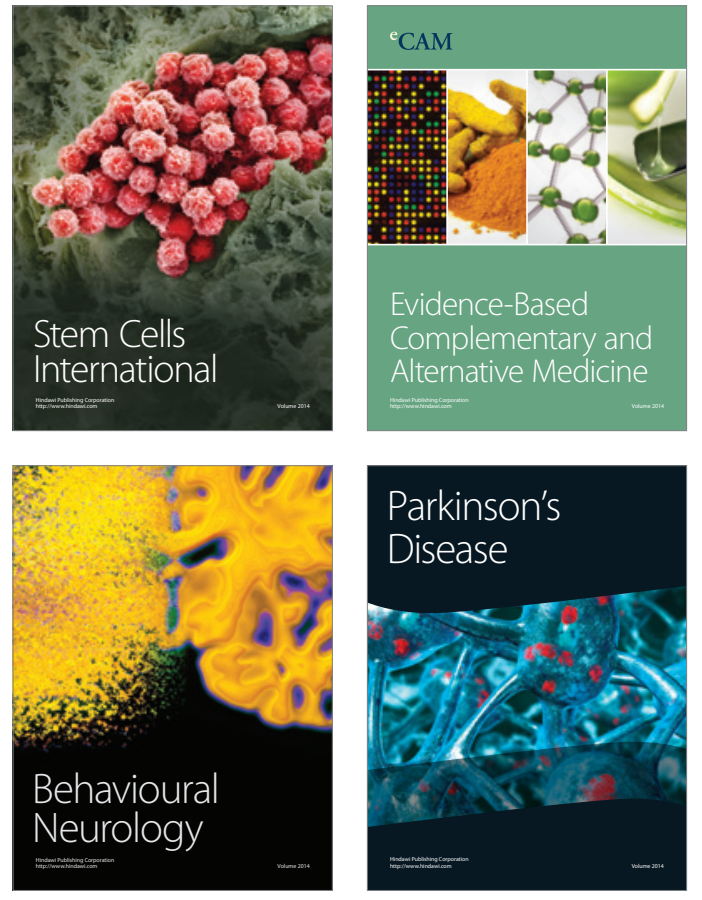
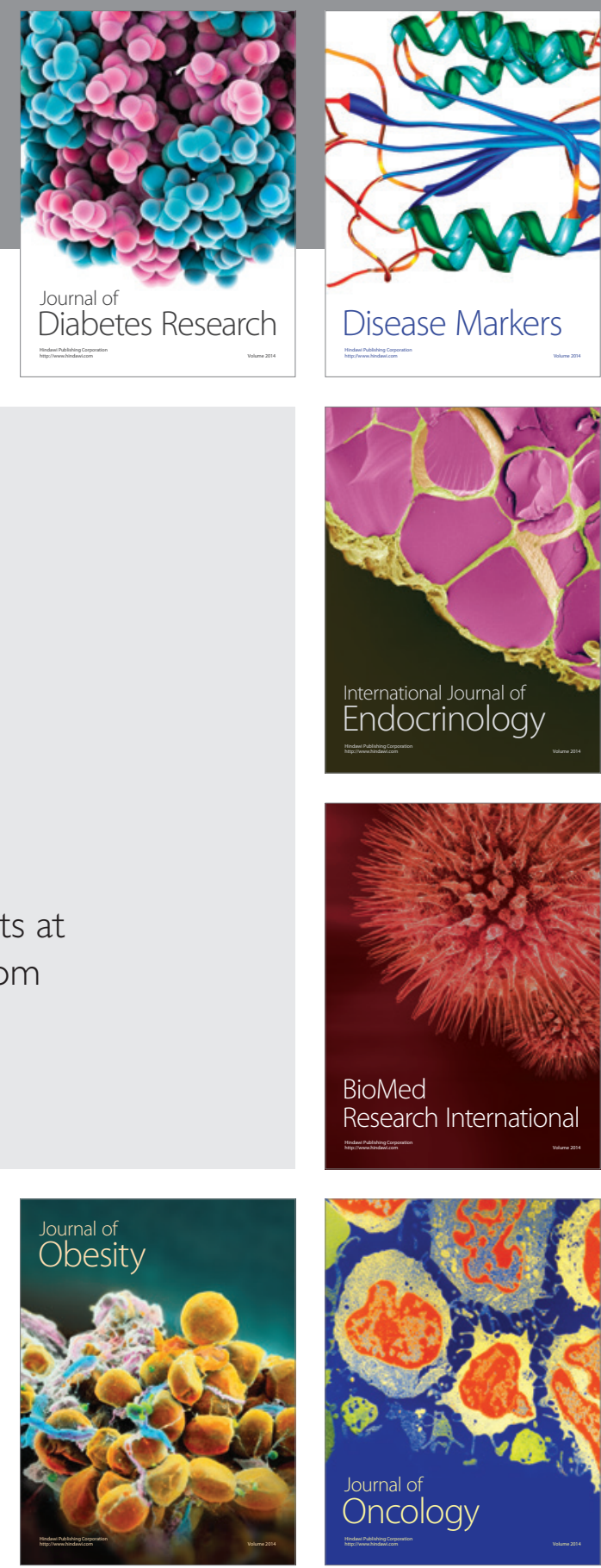

Disease Markers
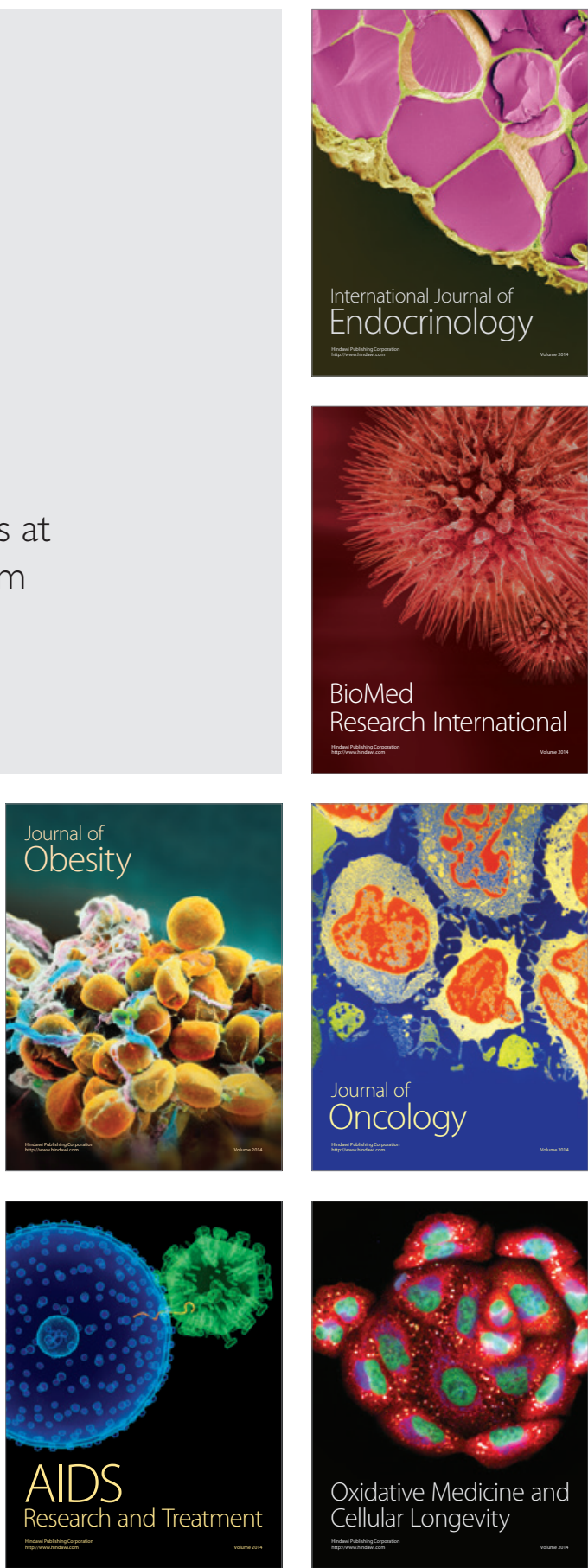